\title{
Optical Identification of X-ray Sources in a High X-ray Flux Sensitivity Area from the RASS
}

\author{
Raúl Mújica \\ INAOE-Tonantzintla, Apdos. Postales 51 y 216 C.P. 72000 Puebla, \\ Pue., México
}

Franz-Josef Zickgraf

Hamburger Sternwarte, Gojenbergsweg 112, 21029 Hamburg, Germany

V. Chavushyan, Y. Juárez, A. Serrano

INAOE-Tonantzintla, Apdos. Postales 51 y 216 C.P. 72000 Puebla, Pue., México

I. Appenzeller, J. Krautter

Landessternwarte-Königstuhl, D-69117 Heidelberg, Germany

\begin{abstract}
.
We have optically identified a complete sub-sample of ROSAT AllSky Survey (RASS) X-ray sources contained in 6 study areas. Of the original $12^{\circ} \times 12^{\circ}$ area near the NEP, only one-quarter was observed until now in order to keep the number of sources to a manageable size. This area is of particular interest because the RASS integration time is about a factor of ten longer than in the other areas and therefore a factor of 3-4 deeper in flux. We have started to observe the RASS sources in the remaining $3 / 4$ of this area. First results are presented.
\end{abstract}

\section{Introduction}

ROSAT detected about 60,000 sources during the All-Sky Survey, i.e., about 1.5 sources per square degree. Complete, spectroscopically identified subsamples of the RASS are extremely important because, first, it is impossible to do spectroscopy on all sources; and second, it is the only way to achieve an unbiased sample of optically identified X-ray sources, in order to carry out statistical studies and to obtain identification criteria to extrapolate towards all X-ray sources.

We have optically identified a complete sub-sample of RASS X-ray sources (Appenzeller et al. 1998, Paper III) contained in 6 study areas. Originally each area measured $12^{\circ} \times 12^{\circ}$ in size. Due to the large number of X-ray sources in two of the six areas, one close to the North Galactic Pole (NGP, area IV) and one near the North Ecliptic Pole (NEP area V), the original sample was reduced in size by observing only part of the latter two areas (Zickgraf et al. 1997, Paper 
II) in order to get a manageable number of sources. The statistical analysis was done by Krautter et al. (1999, Paper IV).

\section{The Area}

The sample was redefined in terms of the minimum fluxes or countrates: $0.01 \mathrm{cts} / \mathrm{s}$ in area $V$ and $0.03 \mathrm{cts} / \mathrm{sec}$ in the other areas. The samples in the NEP and NGP were also reduced in size to smaller sub-areas. The resulting areas were called $\mathrm{Va}$ (western quarter of field V) and IVac (western half of area IV). Area Va was later extended to the east to obtain a field of $6.2^{\circ} \times 6^{\circ}$. Area $\mathrm{V}$ contained 671 sources; 183 were included in area Va. The goal of the present project is to identify the $\mathrm{x}$-ray sources in the remaining $3 / 4$ of the original area, that contains $\sim 300$ sources with countrate $\geq 0.01 \mathrm{cts} / \mathrm{sec}$, following the same ID criteria as in the first part of the project (see Paper II).

\section{Observations}

The observations were carried out at the $2.1 \mathrm{~m}$ telescope of the Guillermo Haro Observatory, operated by INAOE. The LFOSC focal reducer was used; it allows direct CCD imaging, filter photometry, and multi-object spectroscopy. A setup covering the spectral range $4200-9000 \AA$ with a dispersion of $8.2 \AA /$ pix was adopted. Usually, all possible counterparts within a circle of about 50" to 60 " radius around the X-ray position were observed.

\section{Preliminary Results}

We started to observe all the sources inside the error circle of the RASS positions in the remaining $3 / 4$ of area $\mathrm{V}$ with the same limiting count-rate $(0.01 \mathrm{cts} / \mathrm{sec})$. Since May 1999 we have observed more than 100 RASS positions, and we obtained in this way more than 300 spectra of candidate optical counterparts. We are following the method for optical identification as explained in detail in Paper II. The summary of new identifications is as follows: 38 stellar counterparts, 28 AGN, 1 Cluster and 2 Multiple. We have another 50 positions already observed, but not analysed or requiring complementary observations.

\section{References}

Appenzeller et al. 1998, ApJS, 117, 319, Paper III

Condon et al. 1998, AJ, 115, 1693

Krautter et al. 1999, A\&A, 350, 743, Paper IV

Stocke et al. 1991, ApJ, 348, 141

Zickgraf et al. 1997, A\&AS, 123, 103, Paper II 